\title{
Filaggrin mutations p.R501X and c.2282del4 in ichthyosis vulgaris
}

\author{
Robert Gruber $^{1,2}$, Andreas R Janecke ${ }^{2}$, Christine Fauth ${ }^{2}$, Gerd Utermann ${ }^{2}$, Peter O Fritsch ${ }^{1}$ \\ and Matthias Schmuth*,1 \\ ${ }^{1}$ Department of Dermatology, Innsbruck Medical University, Innsbruck, Austria; ${ }^{2}$ Department of Medical Genetics, \\ Molecular and Clinical Pharmacology, Innsbruck Medical University, Innsbruck, Austria
}

Ichthyosis vulgaris (IV) is the most common hereditary disorder of cornification in humans, characterized by generalized fine scaling of the skin, palmar hyperlinearity with or without keratosis pilaris and atopy. Recently, the molecular basis of IV was ascribed to loss-of-function mutations in the gene encoding filaggrin ( $F L G)$, namely p.R501X and c.2282del4. Homozygotes and compound heterozygotes were severely affected whereas heterozygotes showed mild disease or were asymptomatic, suggesting semidominant inheritance with incomplete penetrance in heterozygotes. We report the presence of $F L G$ mutations in 15 out of 21 IV patients with a marked generalized scaling phenotype, including eight affected members of a four-generation family. In this group of patients not only homozygous and compound heterozygous, but also heterozygous patients for p.R501X and c.2282del4 display a pronounced phenotype, whereas in none of six individuals these two mutations were detectable despite decreased filaggrin expression on immunohistochemistry in two patients, indicating that other mutations in $F L G$ and/or in other genes remain to be identified. In contrast, two additional p.R501X heterozygotes from the extended family are asymptomatic. In a control population from west-Austria a combined p.R501X and c.2282del4 carrier frequency of 6/110 (5.45\%) was observed. We confirm that these FLG variants are common, but our results point to the existence of additional modifiers. European Journal of Human Genetics (2007) 15,179-184. doi:10.1038/sj.ejhg.5201742; published online 13 December 2006

Keywords: cornification; differentiation; desquamation; scaling; barrier; keratinocyte; skin

\section{Introduction}

Ichthyoses are a heterogenous group of inherited scaling disorders caused by abnormal cornification. Of these, ichthyosis vulgaris (IV, OMIM 146700) is the most common entity with a reported incidence between 1:250 and 1:5300. ${ }^{1-4}$ Individuals with IV are characterized by a life-long history of generalized fine scaling that is accentuated on the extensor surfaces of the extremities and

\footnotetext{
*Correspondence: Professor M Schmuth, Department of Dermatology, Innsbruck Medical University, Anichstraße 35, A-6020 Innsbruck, Austria. Tel: + 43512504 22971; Fax: + 43512504 22990;

E-mail: matthias.schmuth@i-med.ac.at

Received 9 May 2006; revised 19 September 2006; accepted 17 October 2006; published online 13 December 2006
}

typically spares the great flexures and the neck. Associated findings include hyperkeratosis and hyperlinearity of the palms ('I-hand') and soles, ${ }^{5}$ keratosis pilaris, ${ }^{6}$ hypohydrosis, ${ }^{7}$ atopic dermatitis (AD), ${ }^{8}$ allergic rhinoconjunctivitis and asthma. ${ }^{7,9}$ Histologically, the epidermis of IV subjects is characterized by hyperkeratosis and an attenuation or absence of the granular layer; ${ }^{10-12}$ however, in approximately $25-50 \%$ of patients diagnosed with IV based on clinical criteria the stratum granulosum is histologically normal. 1,13,14 Abnormal keratohyalin granules in the granular layer have been observed on electron microscopy $^{15}$ and decreased epidermal (pro)filaggrin expression has been described as a hallmark of the disease. ${ }^{13,16}$ Recently, two loss-of-function mutations in exon 3 of the gene encoding filaggrin $(F L G)$ have been reported to cause 
the skin abnormalities in IV. $^{17}$ These mutations were described to be inherited in a semidominant pattern with heterozygotes presenting a moderate phenotype with $90 \%$ penetrance and homozygotes and compound heterozygotes showing a severe phenotype. ${ }^{17}$ These findings prompted us to evaluate a cohort of Central European IV patients including an extended multi-generation family for mutations in FLG and the corresponding clinical phenotype.

\section{Subjects and methods}

\section{Affected individuals and phenotypes}

Thirteen unrelated patients with IV as well as eight affected and 16 unaffected members of an extended four-generation family (Figure 1) were included in this study. With the exception of two individuals of Turkish and Indian ancestry, respectively, the patients were of Austrian and Dutch descent (Table 1). Phenotypes were determined by thorough physical examination by two experienced dermatologists. After obtaining written informed consent (protocol approved by Innsbruck Medical University Ethics Committee on Human Research and carried out in accordance with the declaration of Helsinki), venous blood samples were collected from IV patients and family members. Skin biopsies were obtained from affected individuals and processed for routine light microscopy by hematoxylin/eosin staining and for immunohistochemistry. To exclude X-linked recessive ichthyosis, male patients were tested for steroid sulfatase (STS) deficiency by both lipid electrophoresis and STS-deletion-fluorescence in situ hybridization (FISH) using the LSI-steroid sulfatase probe (Vysis, Abbott, Vienna, Austria). ${ }^{18}$ DNA samples from 110 randomly selected, anonymous and unrelated healthy blood donors were subjected to mutation screening as described below.

\section{Immunohistochemistry}

For immunohistochemistry, we used the filaggrin mouse monoclonal antibody of Novocastra Laboratories Ltd (Newcastle upon Tyne, UK) in a working dilution of 1:25 and the high-temperature antigen unmasking technique according to the manufacturer's instructions.

\section{Molecular analysis}

Genomic DNA was extracted from EDTA blood samples by using the QIAamp DNA Mini Kit (Qiagen, Vienna, Austria) or the GenoM48 automated extractor (Qiagen, Vienna, Austria) according to the protocols of the manufacturer.

For the analysis of the published p.R501X (c.1501C > T) mutation localized in exon 3 of the FLG gene, genomic DNA was amplified by using Promega PCR buffer, Promega Taq polymerase mix and the published primer pair FilH1F3/RPT1P6 ${ }^{17}$ using the PTC-100 Peltier Thermal Cycler (MJ Research, USA). PCR conditions were as follows: $94^{\circ} \mathrm{C}$ for $5 \mathrm{~min} ; 35$ cycles of $94^{\circ} \mathrm{C}$ for $40 \mathrm{~s}, 58^{\circ} \mathrm{C}$ for $1 \mathrm{~min}$, $72^{\circ} \mathrm{C}$ for $1 \mathrm{~min} 30 \mathrm{~s}$; final extension step at $72^{\circ} \mathrm{C}$ for $7 \mathrm{~min}$. The p.R501X mutation creates a Hin1II (NlaIII) restriction site, and all samples were screened by restriction digest (Hin1II, Fermentas) of PCR products $\left(3 \mathrm{~h}\right.$ at $\left.37^{\circ} \mathrm{C}\right)$ followed by $3 \%$ agarose gel electrophoresis. Amplified PCR products from three different index patients were also resolved on $1.5 \%$ agarose gels, purified by Ultrafree-DA (www.millipore. com) and sequenced using the Big Dye Terminator protocol.

Similarly, for analysis of the c.2282del4 mutation localized in exon 3 genomic DNA was amplified by using Promega PCR buffer, Promega Taq polymerase mix and the

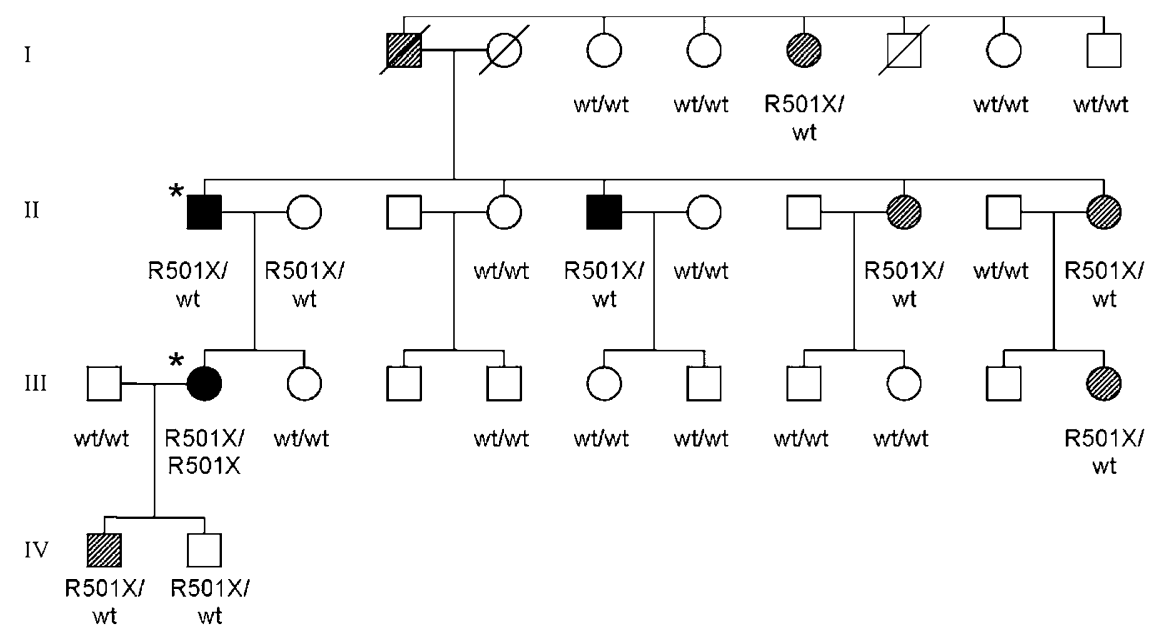

Figure 1 Pedigree of a four-generation IV family. Blackened symbols refer to a severe IV presentation, cross-hatched symbols to a mild IV presentation and open symbols represent unaffected individuals. Genotypes are shown for those family members who underwent testing. The wildtype (wt) symbol refers to the absence of the FLG mutations p.R501X and c.2282del4 of FLG and does not exclude other sequence changes in FLG. Individuals with atopic disease associated with IV are marked with an asterisk. 
Table 1 Genotype-phenotype correlation in 13 IV patients as well as eight affected and 16 unaffected family members of an extended family with IV

\begin{tabular}{|c|c|c|c|}
\hline Individual & Ancestry & $\begin{array}{l}\text { Severity of } \\
\text { phenotype }\end{array}$ & FLG genotype \\
\hline \multicolumn{4}{|c|}{ Individual patients } \\
\hline 1 & Austrian & ++ & R501X/wt \\
\hline 2 & Austrian & ++ & R501X/2282del4 \\
\hline 3 & Austrian & ++ & R501X/2282del4 \\
\hline 4 & Austrian & ++ & $\begin{array}{l}\text { 2282del4/ } \\
\text { 2282del4 }\end{array}$ \\
\hline 5 & Austrian & ++ & R501X/wt \\
\hline 6 & Austrian & ++ & R501X/wt \\
\hline 7 & Austrian & $++^{*}$ & wt/wt \\
\hline 8 & Turkish & $++^{*}$ & wt/wt \\
\hline 9 & Austrian & + & 2282del4/wt \\
\hline 10 & Austrian & $t^{*}$ & $w t / w t$ \\
\hline 11 & Austrian & + & wt/wt \\
\hline 12 & Austrian & $t^{*}$ & wt/wt \\
\hline 13 & Indian & + & wt/wt \\
\hline \multicolumn{4}{|c|}{ Extended family patients } \\
\hline 14 & Dutch & $++^{*}$ & R501X/wt \\
\hline 15 & Dutch & $++^{*}$ & R501X/R501X \\
\hline 16 & Dutch & ++ & R501X/wt \\
\hline 17 & Dutch & + & R501X/wt \\
\hline 18 & Dutch & + & $\mathrm{R} 501 \mathrm{X} / \mathrm{wt}$ \\
\hline 19 & Dutch & + & R501X/wt \\
\hline 20 & Dutch & + & $\mathrm{R} 501 \mathrm{X} / \mathrm{wt}$ \\
\hline 21 & $\begin{array}{l}\text { Dutch/ } \\
\text { Australian }\end{array}$ & + & R501X/wt \\
\hline \multicolumn{4}{|c|}{ Extended family healthy persons } \\
\hline 1 & $\begin{array}{l}\text { Dutch/ } \\
\text { Australian }\end{array}$ & - & R501X/wt \\
\hline 2 & Dutch & - & R501X/wt \\
\hline 3 & Dutch/Swiss & - & $w t / w t$ \\
\hline 4 & Australian & - & wt/wt \\
\hline $5-16$ & Dutch & - & wt/wt \\
\hline
\end{tabular}

' $t$ ' denotes individuals with mild IV phenotype, that is, fine scaling primarily on the extensor surfaces of the extremities.

'++' denotes patients with severe IV phenotype, that is, generalized fine to coarse scaling and marked palmoplantar keratoderma. Individuals with concurrent atopic dermatitis, asthma and/or allergic rhinitis are marked with an asterisk.

published primer pair RPT1P7/RPT2P $1{ }^{17}$ using the following PCR settings: $94^{\circ} \mathrm{C}$ for $5 \mathrm{~min}$; 35 cycles of $94^{\circ} \mathrm{C}$ for $40 \mathrm{~s}, 57^{\circ} \mathrm{C}$ for $1 \mathrm{~min}, 72^{\circ} \mathrm{C}$ for $2 \mathrm{~min}$; final extension step at $72^{\circ} \mathrm{C}$ for $7 \mathrm{~min}$. The c.2282del 4 mutation creates a new AdeI (DraIII) restriction site, and the samples were screened by restriction digest of PCR products using $5 \mathrm{U}$ AdeI for $3 \mathrm{~h}$ at $37^{\circ} \mathrm{C}$ and $2.5 \%$ agarose gel electrophoresis. Amplified PCR products from three probands were also electrophoresed on $1.5 \%$ agarose gels, purified by Ultrafree-DA (www.millipore. com) and sequenced using the Big Dye Terminator protocol to confirm the mutation.

\section{Results}

After excluding X-linked recessive ichthyosis (OMIM $+308100)$ in all male individuals by lack of accelerated low-density lipoprotein migration on serum lipid

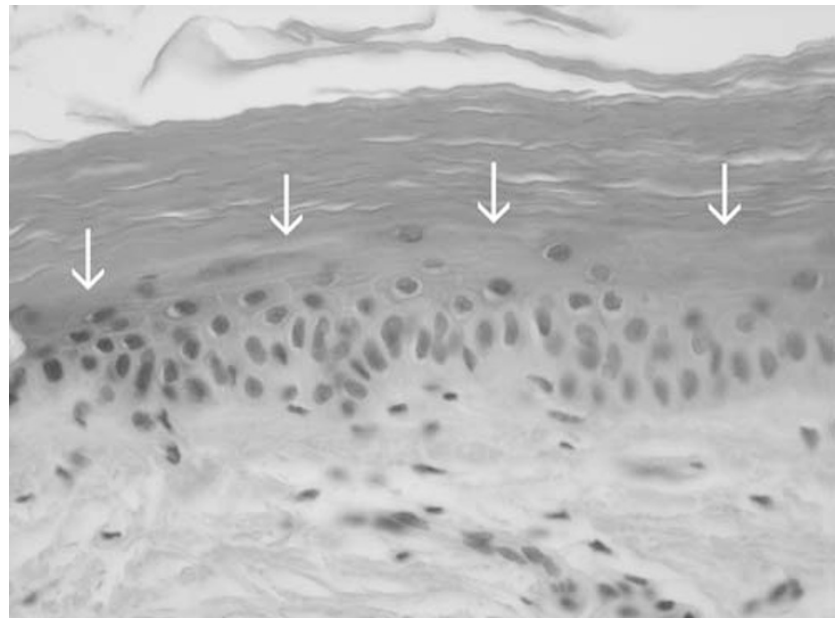

Figure 2 Histology of IV with compact hyperkeratosis and absence of a granular layer (arrows). Hematoxylin- and eosin-stained paraffin section. Magnification $\times 400$.

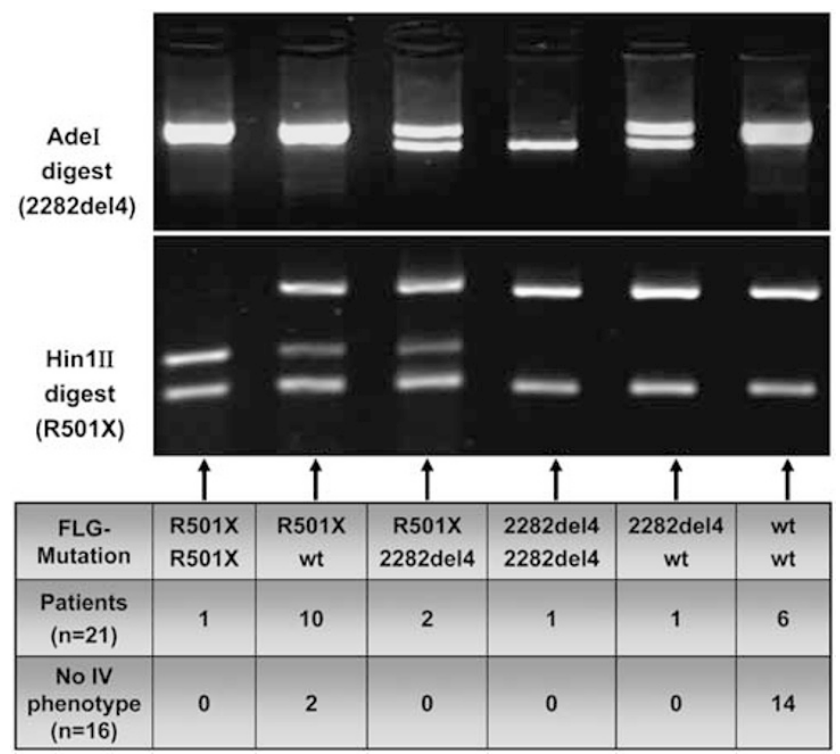

Figure 3 Screening of IV patients for FLG mutations p.R501X and c.2282del4. The p.R501X mutation creates an additional restriction site for Hin1II, the c.2282del4 mutation creates a new restriction site for Adel.

electrophoresis and exclusion of a microdeletion within the STS gene (Xp22. 3) by FISH, we assessed the severity of the IV phenotype of thirteen patients and an extended family with eight affected family members (Figure 1) by clinical examination. There was considerable variation in the degree of generalization and in the intensity of scaling (Table 1). Although the skin of 10 patients was characterized by fine, whitish scaling primarily on the extensor surfaces of the extremities, the other 11 patients showed a severe expression of IV with generalized fine to coarse scaling and marked palmoplantar keratoderma. Six 
patients (29\%) had atopic dermatitis (erythematous, scaly plaques) with or without asthma and/or allergic rhinitis, of whom two carried FLG mutations. Light microscopy of skin biopsies from members of the extended IV pedigree with the pronounced IV-phenotype all revealed absence of the granular layer regardless of body site (Figure 2).

Next, we screened our cohort of patients for the two reported loss-of-function mutations in exon 3 of the FLG
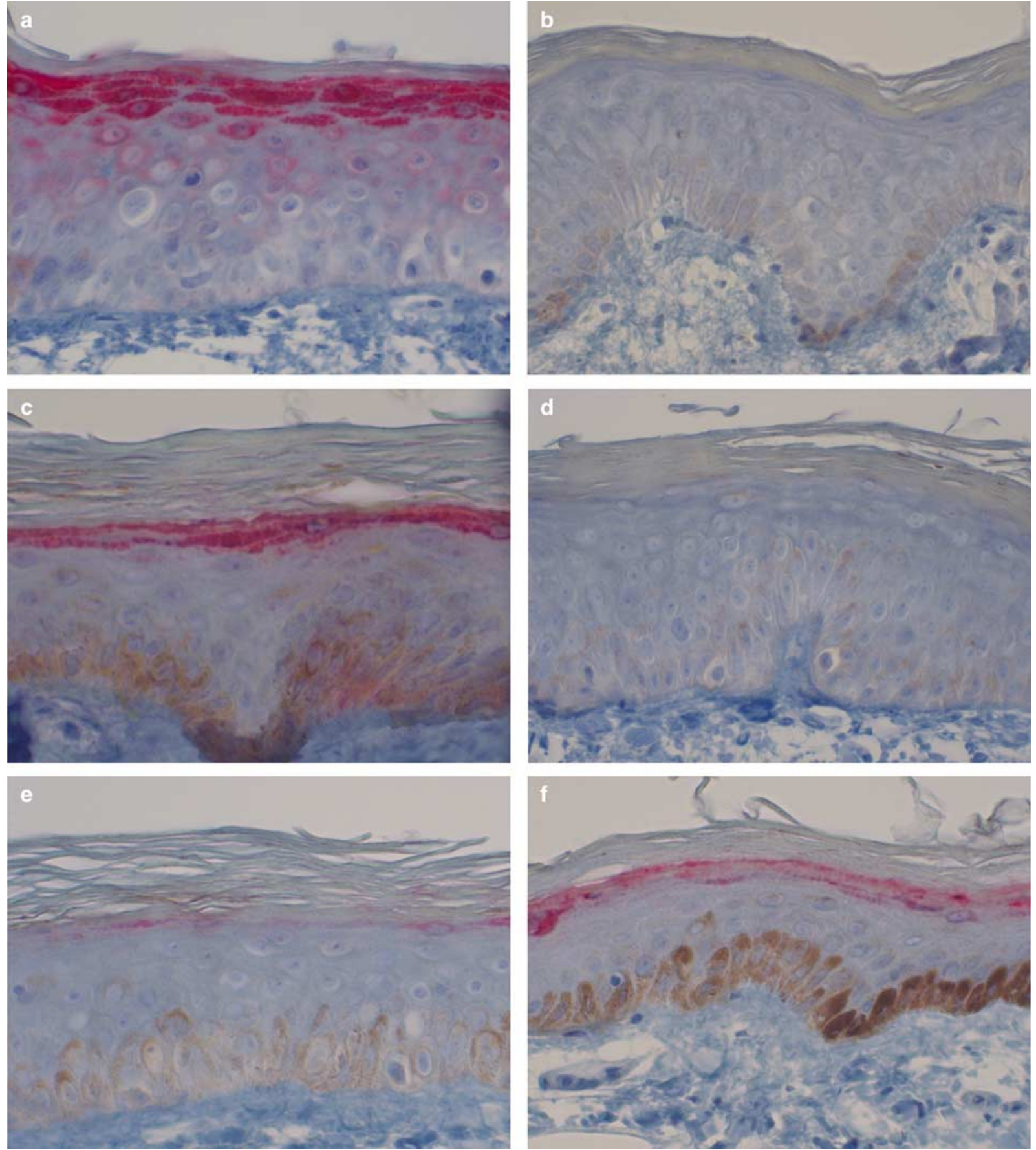

Figure 4 Immunohistochemistry using an antibody directed against the C-terminal filaggrin repeats. (a) Healthy control wild-type (wt) for p.R501X and c.2282del4, exhibiting FLG staining of the granular layer. (b) Compound heterozygous lacking FLG expression. (c) Heterozygous showing decreased $F L G$ expression. (d) Heterozygous lacking $F L G$ expression. (e, f) IV patients with genotypes wt/wt, however, exhibiting variable degrees of $F L G$ expression. Magnification $\times 400$. 
gene, namely p.R501X and c.2282del4 by using restriction enzyme digestion (Figure 3). Of 13 unrelated patients with generalized scaling, three were homozygous or compound heterozygous, and four were heterozygous for one of the mutations. Six IV patients showed neither mutation, but showed the clinical signs of IV and two of them exhibited variable degrees of decreased FLG expression on immunohistochemistry (Figure 4e and f). Furthermore, among eight affected relatives of the extended family, one was homozygous and seven individuals were heterozygous for p.R501X. Within this pedigree, two of 16 family members with no discernible IV phenotype (Figure 1) were found to be heterozygous for p.R501X, corresponding to a penetrance of this mutation of $83 \%$. Finally, in a panel of control samples we observed a carrier frequency of $3 / 110(2.7 \%)$ for the variant p.R501X and $3 / 110(2.7 \%)$ for the variant c.2282del4, respectively.

\section{Discussion}

Recently, the FLG mutations p.R501X or c.2282del4 were identified in all of seven unrelated families and eight sporadic IV patients of Irish, Scottish and EuropeanAmerican origin. ${ }^{17}$ We identified six p.R501X and five c.2282del4 alleles in eight of 14 unrelated IV patients, confirming that FLG mutations are a major cause of IV in patients from Central Europe (Austria and The Netherlands) (Table 1).

A semidominant mode of inheritance of IV was exemplified by multiple examples of p.R501X heterozygous patients with very mild presentation as well as by p.R501X homozygotes and p.R501X/c.2282del4 compound heterozygotes with the full ichthyosis vulgaris phenotype. ${ }^{17}$ Yet, in our IV cohort five patients heterozygous for p.R501X displayed a pronounced ichthyosis phenotype. Conversely, six patients showed neither p.R501X nor c.2282del4 (Table 1) despite generalized scaling and although two of them showed diminished filaggrin expression on immunohistochemistry (Figure $4 \mathrm{e}$ and f). Together, these results raise the possibility of additional allelic or non-allelic mutations in these patients. However, FLG sequencing in the five heterozygous patients and in the two patients of non-European ancestry is slowed by difficulties owing to the length and repetitive nature of this gene. ${ }^{17}$ Alternatively, it is also possible that other cornified envelope proteins, encoded adjacent to $F L G$ in the epidermal differentiation complex including filaggrin 2 , trichohyalin, hornulin, repetin or cornulin harbor mutations in these patients. Finally, the generalized scaling phenotype in these patients could result from variants in independent genes outside the epidermal differentiation complex reminiscent of the genetic heterogeneity in autosomal recessive ichthyoses.
Based on our data, the penetrance of IV in heterozygotes can be estimated at $83 \%$, comparable to the $90 \%$ stated by Smith et $a l^{17}$ on the basis of their seven families; however, these penetrance estimates derive from selected families and may be biased towards higher values.

The association of IV with atopic diathesis is well established; one-third to one-half of patients with IV show concurrent atopic disease, ${ }^{8,19}$ and at least $8 \%$ of patients with $\mathrm{AD}$ have classic features of IV. In the present cohort, six of 21 patients (29\%) had concurrent atopic dermatitis and two of them carried FLG mutations. Very recently a combined p.R501X and c.2282del4 allele frequency of 0.330 was observed in a cohort of 52 children with $\mathrm{AD},{ }^{20}$ indicating a pathophysiological role of filaggrin defects in this disease.

p.R501X and c.2282del4 allele frequencies in Irish, Scottish and European American populations of 0.041 and $0.005,0.021$ and 0.012 and 0.024 and 0.011, respectively, were reported by Smith et $a l^{17}$. Whereas Palmer et $\mathrm{al}^{20}$ reported allele frequencies of 0.029 and 0.019 in a population of 1008 Scottish schoolchildren, we observed frequencies of 0.014 for each allele and a combined allele frequency of 0.027 among Austrian controls. Whether these high mutation frequencies are owing to founder mutations or recurrent de novo mutations, for example, the p.R501X mutation concerns a CpG site, will be addressed by haplotype studies in patients from different populations.

\section{Acknowledgements}

We dedicate this work to Dr Mary Williams who has devoted much of her professional live to the care of patients with ichthyoses. We are grateful to all patients and family members participating in this study for their contribution and commitment. This study was supported by research grants from the Medical Research Fund of the Innsbruck Medical Center (MFF no. 71 and 153) and the Österreichische Nationalbank (ÖNB no. 10822). We are indebted to Professor Christine Heufler for experimental advice and Susanne Neyer and Birgit Moser for technical assistance.

\section{References}

1 Wells RS, Kerr CB: Clinical features of autosomal dominant and sex-linked ichthyosis in an English population. BMJ 1966; 1: 947-950.

2 Al-Zayir AA, Al-Amro Alakloby OM: Primary hereditary ichthyoses in the Eastern Province of Saudi Arabia. Int J Dermatol 2004; 43: 415-419.

3 Okano M, Kitano Y, Yoshikawa K, Nakamura T, Matsuzawa Y, Yuasa T: X-linked ichthyosis and ichthyosis vulgaris: comparison of their clinical features based on biochemical analysis. $\mathrm{Br} J$ Dermatol 1988; 119: 777-783.

4 Cuevas-Covarrubias SA, Diaz-Zagoya JC, Rivera-Vega MR et al: Higher prevalence of $\mathrm{X}$-linked ichthyosis vs ichthyosis vulgaris in Mexico. Int J Dermatol 1999; 38: 555-556.

5 Uehara M, Hayashi S: Hyperlinear palms: association with ichthyosis and atopic dermatitis. Arch Dermatol 1981; 117: 490-491. 
6 Mevorah B, Marazzi A, Frenk E: The prevalence of accentuated palmoplantar markings and keratosis pilaris in atopic dermatitis, autosomal dominant ichthyosis and control dermatological patients. Br J Dermatol 1985; 112: 679-685.

7 Shwayder T, Ott F: All about ichthyosis. Pediatr Clin North Am 1991; 38: 835-857.

8 Rabinowitz LG, Esterly NB: Atopic dermatitis and ichthyosis vulgaris. Pediatr Rev 1994; 15: 220-226; quiz 226.

9 Kuokkanen K: Ichthyosis vulgaris. A clinical and histopathological study of patients and their close relatives in the autosomal dominant and sex-linked forms of the disease. Acta Derm Venereol Suppl (Stockholm) 1969; 62: 1-72.

10 Erickson L, Kahn G: The granular layer thickness in atopy and ichthyosis vulgaris. J Invest Dermatol 1970; 54: 11-12.

11 Hofbauer M, Schnyder UW: Differential diagnosis of autosomaldominant ichthyosis vulgaris and X-chromosomal ichthyosis. Hautarzt 1974; 25: 319-325.

12 Traupe H, Happle R: Clinical features and genetics of the ichthyosis vulgaris group. Fortschr Med 1980; 98: 1809-1815.

13 Fleckman P, Brumbaugh S: Absence of the granular layer and keratohyalin define a morphologically distinct subset of individuals with ichthyosis vulgaris. Exp Dermatol 2002; 11: $327-336$.
14 Fitch N, Segool R, Ferenczy A, Cohen H: Dominant ichthyosis vulgaris with an ultrastructurally normal granular layer. Clin Genet 1976; 9: 71-76.

15 Anton-Lamprecht I, Hofbauer M: Ultrastructural distinction of autosomal dominant ichthyosis vulgaris and $\mathrm{X}$-linked recessive ichthyosis. Humangenetik 1972; 15: 261-264.

16 Sybert VP, Dale BA, Holbrook KA: Ichthyosis vulgaris: identification of a defect in synthesis of filaggrin correlated with an absence of keratohyaline granules. J Invest Dermatol 1985; 84: 191-194.

17 Smith FJ, Irvine AD, Terron-Kwiatkowski A et al: Loss-of-function mutations in the gene encoding filaggrin cause ichthyosis vulgaris. Nat Genet 2006; 38: 337-342.

18 Bonifas JM, Morley BJ, Oakey RE, Kan YW, Epstein Jr EH: Cloning of a cDNA for steroid sulfatase: frequent occurrence of gene deletions in patients with recessive $\mathrm{X}$ chromosome-linked ichthyosis. Proc Natl Acad Sci USA 1987; 84: 9248-9251.

19 Fartasch M, Diepgen TL, Hornstein OP: Atopic dermatitisichthyosis vulgaris-hyperlinear palms-an ultrastructural study. Dermatologica 1989; 178: 202-205.

20 Palmer CN, Irvine AD, Terron-Kwiatkowski A et al: Common lossof-function variants of the epidermal barrier protein filaggrin are a major predisposing factor for atopic dermatitis. Nat Genet 2006; 38: $441-446$ 